\title{
Intraoperative photodynamic diagnosis of lymph node metastasis in esophageal cancer patients using 5-aminolevulinic acid
}

\author{
MASAAKI MOTOORI ${ }^{1}$, MASAHIKO YANO ${ }^{1}$, KOJI TANAKA ${ }^{1}$, KENTARO KISHI ${ }^{1}$, HIDENORI TAKAHASHI ${ }^{1}$, \\ MASAHIRO INOUE ${ }^{2}$, TAKURO SAITO ${ }^{1}$, KEIJIRO SUGIMURA ${ }^{1}$, YOSHIYUKI FUJIWARA ${ }^{1}$, \\ OSAMU ISHIKAWA ${ }^{1}$ and MASATO SAKON ${ }^{1}$
}

Departments of ${ }^{1}$ Surgery and ${ }^{2}$ Biochemistry, Osaka Medical Center for Cancer and Cardiovascular Diseases, Osaka 537-8511, Japan

Received November 10, 2014; Accepted August 5, 2015

DOI: $10.3892 / \mathrm{ol} .2015 .3685$

\begin{abstract}
Lymph node metastasis is the strongest prognostic factor in esophageal cancer patients who have undergone esophagectomy. The accurate diagnosis of lymph node metastasis is important, but the pre-operative diagnostic accuracy is poor. The intraoperative diagnosis based on histopathological examination of frozen tissue specimens is complicated and time-consuming. Therefore, the establishment of a simple and rapid intraoperative diagnostic method is essential. Exogenous application of 5-aminolevulinic acid (ALA) causes a selective accumulation of protoporphyrin IX, which is a fluorescent substrate, in cancer cells. The present study evaluated the feasibility of photodynamic diagnosis using ALA (ALA-PDD) for lymph node metastasis in esophageal cancer. A total of 292 lymph nodes were analyzed from 8 esophageal squamous cell cancer patients treated with esophagectomy. The patients were administered ALA orally prior to surgery. Excised lymph nodes were cut in half and examined by spectrometer. The diagnostic results of ALA-PDD were compared to those of the histopathological examination. Among the 292 lymph nodes, 19 nodes $(6.5 \%)$ were histologically metastatic and 21 nodes $(7.2 \%)$ were PDD-positive. The sensitivity and specificity of ALA-PDD were $84.2 \%(16 / 19)$ and $98.2 \%$ (268/273), respectively. The area of cancer nests of the PDD-negative lymph nodes was $<2 \mathrm{~mm}^{2}$. Metastatic lymph nodes, including cancer nests $>4 \mathrm{~mm}^{2}$, were correctly diagnosed by ALA-PDD. In conclusion, this study demonstrated that ALA-PDD of lymph node metastasis in patients with esophageal cancer is feasible. Further investigation would make this method a simple and rapid intraoperative diagnostic tool.
\end{abstract}

Correspondence to: Dr Masaaki Motoori, Department of Surgery, Osaka Medical Center for Cancer and Cardiovascular Diseases, 1-3-3 Nakamichi, Higashinari-ku, Osaka 537-8511, Japan

E-mail: m-moto@fg8.so-net.ne.jp

Key words: aminolevulinic acid, esophageal cancer, intraoperative diagnosis, lymph node metastasis, photodynamic diagnosis

\section{Introduction}

Surgical resection is currently the optimal curative treatment in esophageal cancer patients without distant metastases. However, even after curative resection by esophagectomy with extended three-field lymphadenectomy, the recurrence of cancer is found in $\sim 50 \%$ of patients (1). Nodal status, including the metastatic lymph node number and extent, is the strongest prognostic factor in esophageal cancer patients who have undergone esophagectomy (1). Accurate assessment of lymph node status is essential. However, pre-operative evaluation of lymph node metastasis is difficult, as smaller lymph nodes cannot be detected by radiographic examination. Indeed, the pre-operative diagnostic accuracy of lymph node metastasis by computed tomography or endoscopic ultrasonography is $60-80 \%$ and remains unsatisfactory $(2,3)$.

The intraoperative diagnosis of lymph node metastasis is currently based on histopathological examination of frozen tissue specimens stained with hematoxylin and eosin. Recently, genetic analysis, such as polymerase chain reaction of molecular markers specific to cancer cells, has been used to detect lymph node metastasis $(4,5)$. However, these methods are complicated and time-consuming, and not suitable for examining a large number of specimens $(4,5)$. Therefore, the establishment of a simple and rapid intraoperative diagnostic method of lymph node metastasis is important.

Photodynamic diagnosis using 5-aminolevulinic acid (ALA-PDD) has recently emerged as a promising technique for detecting cancer nests. ALA is the natural precursor of the heme pathway. Exogenous application of ALA causes a selective accumulation of a heme precursor, protoporphyrin IX (PPIX), in cancer cells due to an increase in porphobilinogen deaminase activity and a decrease in ferrochelatase activity. PPIX is a fluorescent substrate that emits a red fluorescence at $\sim 635 \mathrm{~nm}$ on blue light excitation (6). ALA-PDD has proven useful in various types of malignancies. In early bladder cancer, the introduction of ALA cystoscopy has increased the recurrence-free survival rate compared with white-light cystoscopy after the transurethral resection of superficial bladder cancer $(7,8)$. In gastric cancer, our previous study reported that staging laparoscopy using ALA improved the detection sensitivity of peritoneal metastasis (9). In malignant 
glioma, intraoperative ALA-induced fluorescence guidance was useful for the complete removal of tumors and improved progression-free survival (10). However, to the best of our knowledge, the usefulness of ALA-PDD in human esophageal cancer has not been investigated. The present study evaluated the feasibility of ALA-PDD of lymph node metastasis in patients with esophageal cancer.

\section{Materials and methods}

Uptake of ALA in a cell line. First, ALA-induced PPIX red fluorescence was confirmed in an esophageal cancer cell line. Human esophageal squamous cell cancer TE-1 cells were cultured in RPMI-1640 medium (Gibco Life Technologies, Tokyo, Japan) in a 6-well plate at a concentration of $140,000 / \mathrm{ml}$ and incubated at $37^{\circ} \mathrm{C}$ in a humidified atmosphere of $5 \% \mathrm{CO}_{2}$ for one night. Next, the medium was changed to RPMI-1640 containing $0,0.25,0.5$ or $1.0 \mathrm{mg} / \mathrm{ml}$ ALA hydrochloride (Cosmo Bio Co., Ltd., Tokyo, Japan), and the cells were incubated at $37^{\circ} \mathrm{C}$ for $60 \mathrm{~min}$. Once the medium had been changed to RPMI-1640 containing no ALA, $5 \mu$ l Hoechst 33342 (Sigma-Aldrich, Tokyo, Japan) was added to each dish. Finally, the TE-1 cells were subjected to observation under a fluorescence microscope (BZ-8100; Keyence, Tokyo, Japan). An OP-66834 BZ fluorescence filter (excitation, $360 \mathrm{~nm}$; emission, $460 \mathrm{~nm}$; dichroic mirror, $400 \mathrm{~nm}$; Keyence) and an OP-66838 BZ fluorescence filter (excitation, $560 \mathrm{~nm}$; emission, $630 \mathrm{~nm}$; dichroic mirror, $595 \mathrm{~nm}$, Keyence) were used for Hoechst and PPIX observations, respectively.

Fluorescence microscopy of metastatic lymph nodes. Second, the study confirmed whether the distribution of ALA-induced PPIX red fluorescence agreed with the presence of cancer nests in apparently metastatic lymph nodes in patients with esophageal squamous cell cancer at Osaka Medical Center for Cancer and Cardiovascular Diseases (Osaka, Japan). Between June 2010 and August 2010, ALA hydrochloride (1 g/body; Cosmo Bio Co., Ltd.) was dissolved in $20 \mathrm{ml}$ of a $5 \%$ glucose solution and orally administered to 2 patients with esophageal squamous cell cancer $3 \mathrm{~h}$ prior to esophagectomy. Patients were protected from direct sunlight for $24 \mathrm{~h}$ to avoid phototoxic reactions. Surgical therapy consisted of en bloc esophagectomy via a right thoracotomy with two- or three-field lymphadenectomy, and reconstruction using the stomach, jejunum or colon. Excised metastatic lymph nodes were immediately embedded in TissueTek OCT medium (Sakura, Tokyo, Japan) and frozen at $-80^{\circ} \mathrm{C}$ for $10 \mathrm{~min}$. In a dark room, 2 cryosections of an $8-\mu \mathrm{m}$ thickness were prepared. One section was stained with hematoxylin and eosin to confirm the presence of cancer. Another section was stained with hematoxylin and Hoechst 33342 (Sigma-Aldrich, Tokyo, Japan). Each section was subjected to observation by fluorescence microscopy (BZ-8100; Keyence). An OP-66834 BZ fluorescence filter (excitation, $360 \mathrm{~nm}$; emission, $460 \mathrm{~nm}$; dichroic mirror, $400 \mathrm{~nm}$; Keyence) and an OP-66838 BZ fluorescence filter (excitation, $560 \mathrm{~nm}$; emission, $630 \mathrm{~nm}$; dichroic mirror, $595 \mathrm{~nm}$; Keyence) were used for Hoechst and PPIX observation, respectively.

Intraoperative PDD of lymph node metastasis. Next, intraoperative PDD of lymph node metastasis was performed in
8 patients with esophageal squamous cell cancer between June 2010 and February 2012. The administration of ALA and surgical treatment were performed as aforementioned. Excised lymph nodes were sliced through the center and subjected to PDD. ALA-induced PPIX fluorescence was analyzed using a spectrometer (VLD-M1; M\&M Co., Ltd., Tokyo, Japan) and its accessory software (BWSpec V3.24; B\&W Tek, Inc., Newark, DE, USA). Lymph nodes were exposed to a laser light that exhibited a peak wavelength of $405 \mathrm{~nm}$, and those which exhibited a peak wavelength of $635 \mathrm{~nm}$ were defined as metastasis-positive on PDD, as shown in Fig. 1. Following PDD, each lymph node was fixed with $10 \%$ formalin and subjected to a routine histopathological examination, which was regarded as the gold standard diagnostic procedure. The study protocol was approved by the Human Ethics Review Committee of Osaka Medical Center for Cancer and Cardiovascular Diseases. Written informed consent was obtained from all patients.

\section{Results}

PPIX fluorescence in a cell line. ALA-induced PPIX red fluorescence was observed in the TE-1 cells incubated with 0.25 , 0.5 and $1.0 \mathrm{mg} / \mathrm{ml} \mathrm{ALA}$, but not in the TE-1 cells incubated without ALA (Fig. 2).

Distribution of PPIX in metastatic lymph nodes. The distribution of ALA-induced PPIX red fluorescence in apparently metastatic lymph nodes was analyzed in 5 patients with esophageal squamous cell cancer. The distribution of red fluorescence was identical to that of the metastatic focus (Fig. 3).

Comparison of PDD of lymph node metastasis with pathological diagnosis. Patient characteristics are shown in Table I. Tumor location, depth of tumor and lymph node metastasis were based on the 7th edition of the Union for International Cancer Control tumor-node-metastasis classification guidelines (11). A total of 3 patients were diagnosed with clinical T2 disease and 5 patients with clinical T3 disease. While 2 patients were clinically node-negative, 6 patients were node-positive. All patients were pathologically node-positive. Of all the 292 lymph nodes, 19 nodes were pathologically metastatic and the remaining 273 nodes were pathologically non-metastatic. Table II shows the comparison of PDD with the pathological diagnosis. The sensitivity and specificity of PDD were $84.2 \%$ (16/19) and 98.2\% (268/273), respectively. Furthermore, PDD was compared with the clinical diagnosis in pathologically metastatic nodes. All the clinically-positive nodes were correctly diagnosed by PDD. Among 10 clinically-negative but pathologically-positive nodes, 7 nodes were diagnosed as positive by PDD and the remaining 3 nodes were diagnosed as negative by PDD (Table III). Fig. 4 shows the area of cancer nests in the pathologically metastatic lymph nodes. The metastasis size of the PDD-negative lymph nodes was $<2 \mathrm{~mm}^{2}$. Metastatic lymph nodes, including cancer nests $>4 \mathrm{~mm}^{2}$, were correctly diagnosed by ALA-PDD.

\section{Discussion}

This is the first study demonstrating that ALA-PDD of lymph node metastasis in patients with esophageal squamous cell 
Table I. Patient characteristics.

\begin{tabular}{|c|c|}
\hline Characteristics & Value \\
\hline \multicolumn{2}{|l|}{ Gender, $\mathrm{n}$} \\
\hline Male/female & $7 / 1$ \\
\hline Median age (range), years & $65(52-78)$ \\
\hline \multicolumn{2}{|l|}{ Tumor location, $\mathrm{n}$} \\
\hline Upper/middle/lower & $2 / 4 / 2$ \\
\hline Depth of tumor, $n$ & $3 / 5$ \\
\hline \multicolumn{2}{|l|}{ Lymph node metastasis, $\mathrm{n}$} \\
\hline $\begin{array}{l}\text { Tumor location, depth of tun } \\
\text { based on the } 7 \text { th edition of the } \\
\text { tumor-node-metastasis classifi }\end{array}$ & $\begin{array}{l}\text { tastasis were } \\
\text { ancer Control }\end{array}$ \\
\hline
\end{tabular}

Table II. Comparison of the PDD of lymph nodes with the pathological diagnosis.

\begin{tabular}{lrrr}
\hline & \multicolumn{2}{c}{ Histology } & \\
\cline { 2 - 3 } ALA-PDD & Positive & Negative & Total \\
\hline Positive & 16 & 5 & 21 \\
Negative & 3 & 268 & 271 \\
Total & 19 & 273 & 292
\end{tabular}

ALA-PDD, photodynamic diagnosis using 5-aminolevulinic acid.

Table III. Comparison of the PDD of lymph nodes with the clinical diagnosis.

\begin{tabular}{lccr}
\hline & \multicolumn{2}{c}{ Clinical diagnosis } & \\
\cline { 2 - 3 } ALA-PDD & Positive & Negative & Total \\
\hline Positive & 3 & 0 & 3 \\
Negative & 7 & 9 & 16 \\
Total & 10 & 9 & 19 \\
\hline
\end{tabular}

ALA-PDD, photodynamic diagnosis using 5-aminolevulinic acid.

cancer is feasible. The present study determined that the time required for diagnosing the metastatic status of one lymph node by ALA-PDD is only a few minutes. When compared with conventional histopathological examination of frozen tissue specimens, ALA-PDD is simple and useful for examining a large number of lymph nodes. ALA is metabolized and excreted within 24-48 h, and the time of shielding from strong light is short compared with other photosensitizers, such as hematoporphyrin and Photofrin. In this study, no adverse events, including cutaneous photosensitization, were

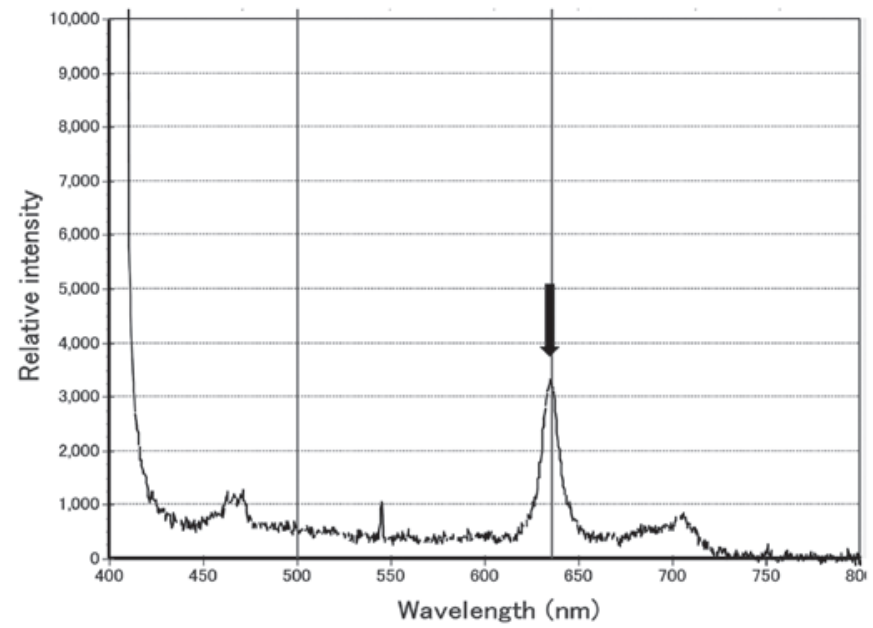

Figure 1. Photodynamic diagnosis (PDD) of lymph nodes using a spectrometer. 5-aminolevulinic acid-induced protoporphyrin IX emits red fluorescence at an $\sim 635$-nm wavelength. Lymph nodes that exhibited a peak wavelength of $635 \mathrm{~nm}$ (arrow) were defined as metastasis-positive on PDD.

observed. Therefore, ALA-PDD is a safe and promising method of intraoperative diagnosis of lymph node metastasis.

In the present study, the excised lymph nodes were sliced prior to performing PDD. Usually, lymph nodes are surrounded by a number of connective tissues. The penetration depth of red light ranges from 0.2 to $2 \mathrm{~cm}$, with a mean depth of $\sim 0.6 \mathrm{~cm}$, and that of blue light is even shallower $(12,13)$. The penetration depth of excitation blue light is too short to reach metastatic lesions buried in tissues. Previously, we performed PDD without cutting the lymph nodes, but the detection rate of PPIX fluorescence was poor (unpublished data). In gastric cancer, Koizumi et al performed fluorescence imaging of the cut surface of lymph nodes (14), as did the present study. Further examination is necessary for intraoperative PDD of lymph node metastasis without excision of the lymph nodes.

In the present study, all the clinically-positive nodes were correctly diagnosed by PDD. False-negative results were observed in 3 clinically-negative nodes. The size of the metastatic focus of these 3 lymph nodes was $<2 \mathrm{~mm}^{2}$ and relatively small. It is considered that the smaller the cancer nest, the smaller the amount of accumulated PPIX. Furthermore, a small cancer nest may be easily affected by photobleaching, a phenomenon whereby the photosensitizer is photochemically destroyed by light. To induce higher intracellular PPIX levels, chemical modifications of ALA, such as esterification with aliphatic alcohols, may be useful for developing a more sensitive method of PDD (15).

Additionally, a number of false-positive cases were observed in the present study. A non-metastatic lymph node was observed, in which PPIX red fluorescence was observed in the marginal sinus by fluorescence microscopy observation (data not shown). This may cause a false-positive result. Koizumi et al reported that they observed some non-metastatic lymph nodes presenting accumulation of PPIX red fluorescence in normal lymphoid follicles (14). This phenomenon may have also occurred in the present study. However, fluorescence microscopy examination was only performed on a small number of lymph nodes. 


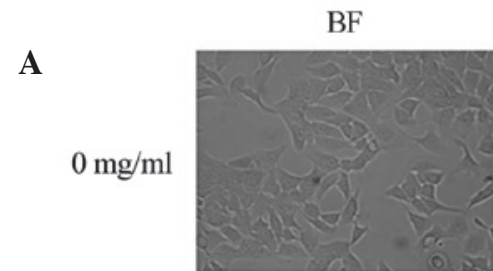

B

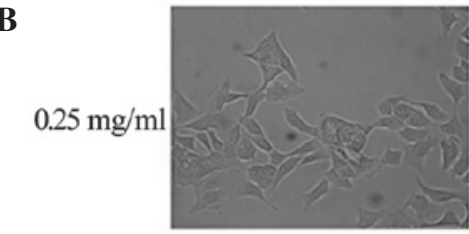

C

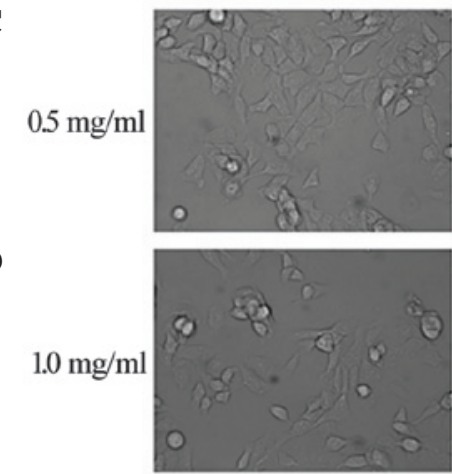

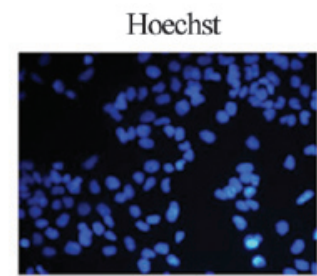
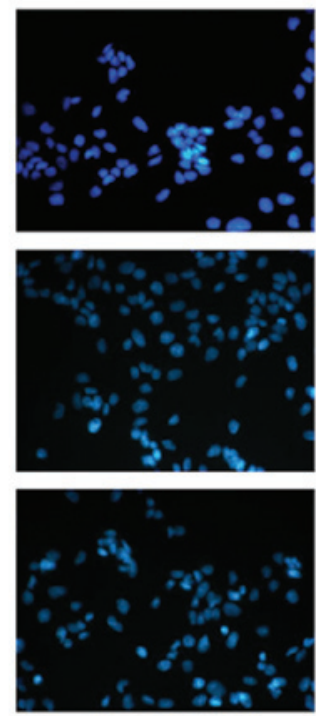
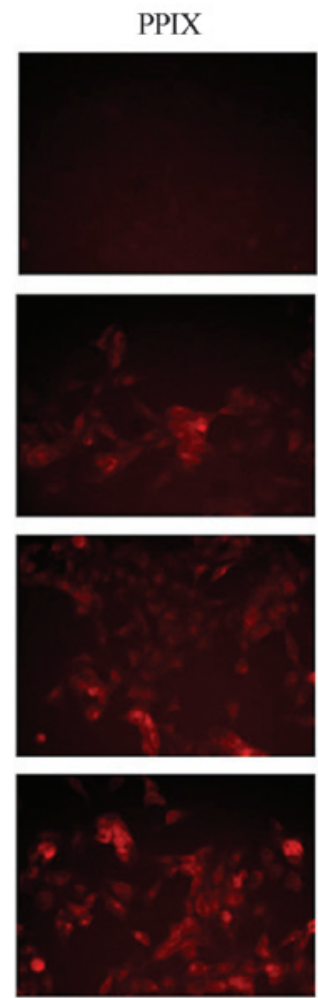
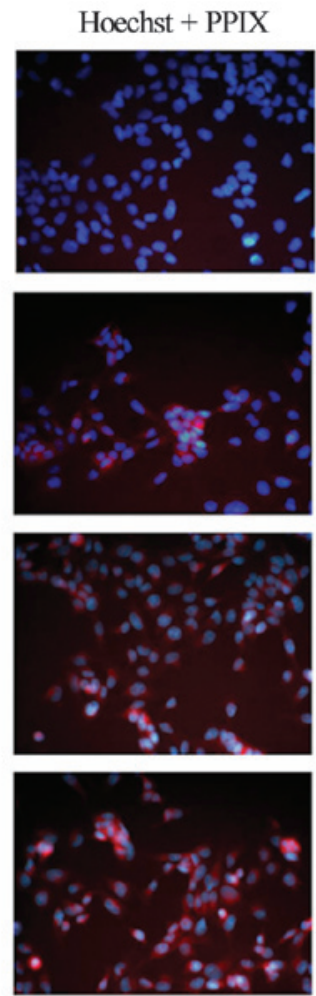

Figure 2. Uptake of ALA in the esophageal squamous cell cancer cell line. ALA-induced PPIX red fluorescence was not observed in TE-1 cells incubated (A) without ALA, but was observed in TE-1 cells incubated with (B) 0.25 , (C) 0.5 and (D) $1.0 \mathrm{mg} / \mathrm{ml}$ ALA. Original magnification, x100. ALA, 5-aminolevulinic acid; BF, buffered formalin; PPIX, protoporphyrin IX.

HE
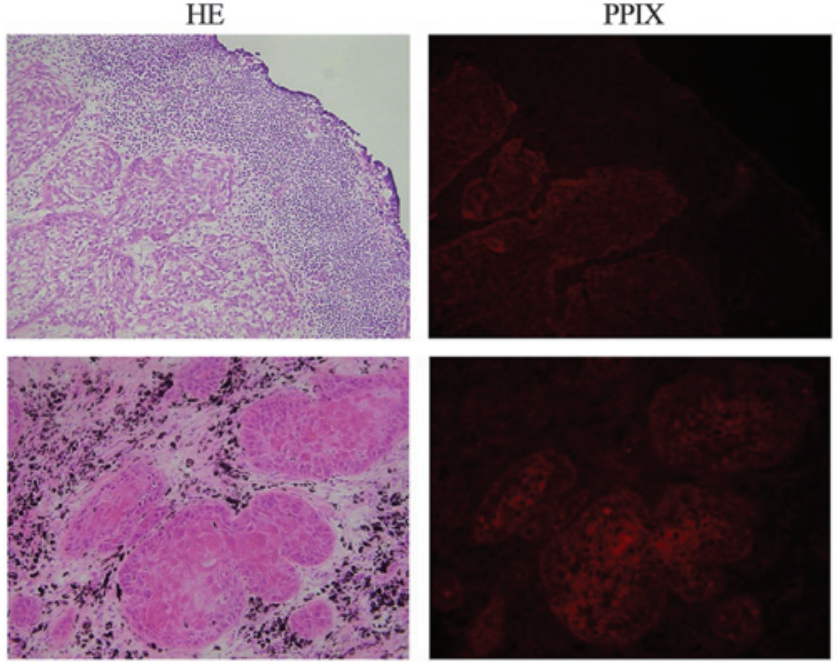

Figure 3. Representative images of microscopic observations of the metastatic lymph nodes. The distribution of 5-aminolevulinic acid-induced protoporphyrin IX red fluorescence was identical to that of the metastatic focus. Original magnification, x100. HE, hematoxylin and eosin; PPIX, protoporphyrin IX.

Previous studies demonstrated that PPIX tends to accumulate at an inflamed site (16). The detailed mechanism of PPIX accumulation in non-cancerous lesions is unclear.

For the further application of ALA-induced fluorescence in esophageal cancer surgery, intraoperative PDD of resection margins, such as in the aorta, trachea and recurrent laryngeal nerve, are considered to be important. Preliminary ALA-PDD

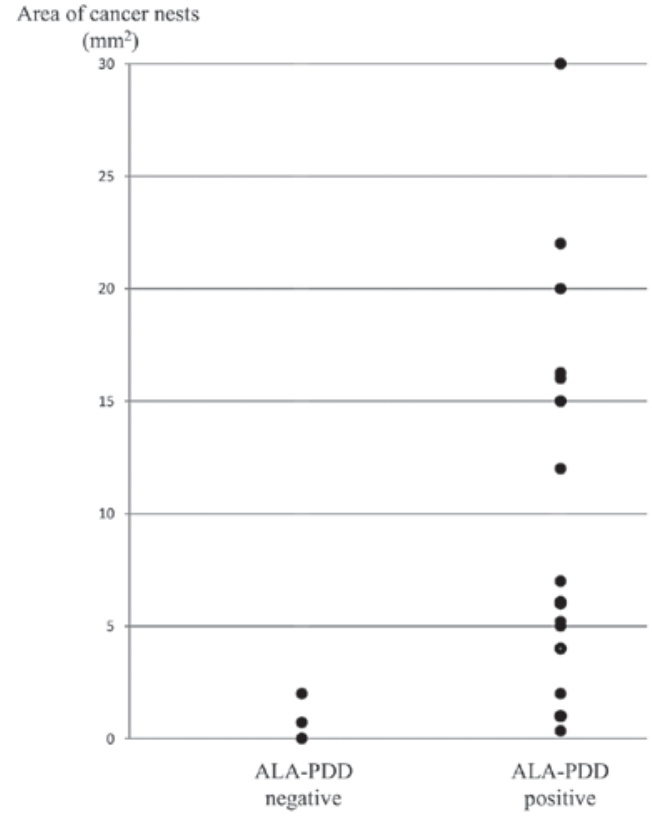

Figure 4. Distribution of the area of cancer nests in the metastatic lymph nodes. ALA-PDD, photodynamic diagnosis using 5-aminolevulinic acid.

was performed in the present study for evaluating remnant cancer in the surgical margins around the trachea, but the evaluation was extremely difficult. One of the problems of evaluating the surgical margin is hemorrhage, as hemoglobin absorbs blue excitation light. Several problems remain to be resolved prior to applying ALA-PDD to the surgical margin of 
esophageal cancer. ALA-induced fluorescence has been used not only for diagnosis but also for treatment. In nodular basal cell carcinoma, PD therapy (PDT) using methyl aminolevulinate cream is effective (17). The complete response rate of clinically assessed lesion clearance did not differ significantly between the PDT group and the surgically-treated group (17). Pech et al reported that the excellent long-term results of ALA-PDT in patients with superficial Barrett's esophageal cancer or high-grade intraepithelial neoplasia and ALA-PDT may be an alternative treatment to esophagectomy and endoscopic resection, particularly in cases with multifocal Barrett's neoplasia (18). In patients with advanced esophageal cancer, intraoperative ALA-PDT for surgical margins where microscopic cancer nests may be left has potential as a novel treatment option.

In conclusion, the present study demonstrated that ALA-PDD of lymph node metastasis in patients with esophageal cancer is feasible. Further investigation would make this method a simple and rapid intraoperative diagnostic tool.

\section{Acknowledgements}

The authors would like to thank Ms. Noriko Kanto for providing expert technical assistance.

\section{References}

1. Akiyama H, Tsurumaru M, Udagawa $H$ and Kajiyama $Y$ : Radical lymph node dissection for cancer of the thoracic esophagus. Ann Surg 220: 364-372, 1994.

2. Wu LF, Wang BZ, Feng JL, Cheng WR, Liu GR, Xu XH and Zheng ZC: Preoperative TN staging of esophageal cancer: Comparison of miniprobe ultrasonography, spiral CT and MRI. World J Gastroenterol 9: 219-224, 2003.

3. Nishimaki T, Tanaka O, Ando N, Ide H, Watanabe H, Shinoda M, Takiyama W, Yamana H, Ishida K, Isono K, et al: Evaluation of the accuracy of preoperative staging in thoracic esophageal cancer. Ann Thorac Surg 68: 2059-2064, 1999.

4. Yoshioka S, Fujiwara Y, Sugita Y, Okada Y, Yano M, Tamura S, Yasuda T, Takiguchi S, Shiozaki H and Monden M: Real-time rapid reverse transcriptase-polymerase chain reaction for intraoperative diagnosis of lymph node micrometastasis: Clinical application for cervical lymph node dissection in esophageal cancers. Surgery 132: 34-40, 2002.

5. Miyata H, Yano M, Doki Y, Yasuda T, Yoshioka S, Sugita Y, Takiguchi S, Fujiwara Y and Monden M: A prospective trial for avoiding cervical lymph node dissection for thoracic esophageal cancers, based on intra-operative genetic diagnosis of micrometastasis in recurrent laryngeal nerve chain nodes. J Surg Oncol 93: 477-484, 2006.

6. Hinnen P, de Rooij FW, van Velthuysen ML, Edixhoven A, van Hillegersberg R, Tilanus HW, Wilson JH and Siersema PD: Biochemical basis of 5-aminolaevulinic acid-induced protoporphyrin IX accumulation: A study in patients with (pre)malignant lesions of the oesophagus. Br J Cancer 78: 679-682, 1998.
7. Daniltchenko DI, Riedl CR, Sachs MD, Koenig F, Daha KL, Pflueger H, Loening SA and Schnorr D: Long-term benefit of 5-aminolevulinic acid fluorescence assisted transurethral resection of superficial bladder cancer: 5-year results of a prospective randomized study. J Urol 174: 2129-2133, 2005.

8. Denzinger S, Burger M, Walter B, Knuechel R, Roessler W, Wieland WF and Filbeck T: Clinically relevant reduction in risk of recurrence of superficial bladder cancer using 5-aminolevulinic acid-induced fluorescence diagnosis: 8-year results of prospective randomized study. Urology 69: 675-679, 2007.

9. Kishi K, Fujiwara Y, Yano M, Inoue M, Miyashiro I, Motoori M, Shingai T, Gotoh K, Takahashi H, Noura S, et al: Staging laparoscopy using ALA-mediated photodynamic diagnosis improves the detection of peritoneal metastases in advanced gastric cancer. J Surg Oncol 106: 294-298, 2012.

10. Stummer W, Pichlmeier U, Meinel T, Wiestler OD, Zanella F and Reulen HJ; ALA-Glioma Study Group: Fluorescence-guided surgery with 5-aminolevulinic acid for resection of malignant glioma: A randomised controlled multicentre phase III trial. Lancet Oncol 7: 392-401, 2006.

11. Sobin LH, Gospodarowicz MK and Wittekind CH: TNM classification of malignant tumors. 7th edition. Wiley-Blackwell, Oxford, UK, 2009.

12. Webber J, Herman N, Kessel D and Fromm D: Current concepts in gastrointestinal photodynamic therapy. Ann Surg 230: 12-23, 1999.

13. Braathen LR, Szeimies RM, Basset-Seguin N, Bissonnette R, Foley P, Pariser D, Roelandts R, Wennberg AM and Morton CA; International Society for Photodynamic Therapy in Dermatology: Guidelines on the use of photodynamic therapy for nonmelanoma skin cancer: an international consensus. International society for photodynamic therapy in dermatology, 2005. J Am Acad Dermatol 56: 125-143, 2007.

14. Koizumi N, Harada Y, Murayama Y, Harada K, Beika M, Yamaoka Y, Dai P, Komatsu S, Kubota T, Ichikawa D, et al: Detection of metastatic lymph nodes using 5-aminolevulinic acid in patients with gastric cancer. Ann Surg Oncol 20: 3541-3548, 2013.

15. Gaullier JM, Berg K, Peng Q, Anholt H, Selbo PK, Ma LW and Moan J: Use of 5-aminolevulinic acid esters to improve photodynamic therapy on cells in culture. Cancer Res 57: 1481-1486, 1997.

16. Messmann H, Knuchel R, Bäumler W, Holstege A and Schölmerich J: Endoscopic fluorescence detection of dysplasia in patients with Barrett's esophagus, ulcerative colitis, or adenomatous polyps after 5-aminolevulinic acid-induced protoporphyrin IX sensitization. Gastrointest Endosc 49: 97-101, 1999.

17. Rhodes LE, de Rie M, Enström Y, Groves R, Morken T, Goulden V, Wong GA, Grob JJ, Varma S and Wolf P: Photodynamic therapy using topical methyl aminolevulinate vs surgery for nodular basal cell carcinoma: results of a multicenter randomized prospective trial. Arch Dermatol 140: 17-23, 2004.

18. Pech O, Gossner L, May A, Rabenstein T, Vieth M, Stolte M, Berres $\mathrm{M}$ and Ell C: Long-term results of photodynamic therapy with 5-aminolevulinic acid for superficial Barrett's cancer and high-grade intraepithelial neoplasia. Gastrointest Endosc 62: 24-30, 2005. 